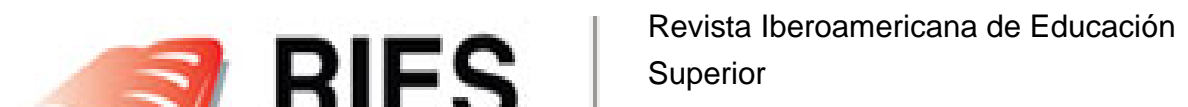 \\ E-ISSN: 2007-2872 \\ Revista lberoamericana de Educación Superior \\ emmaro@unam.mx \\ Instituto de Investigaciones sobre la \\ Universidad y la Educación \\ México
}

Cuevas-Novoa, Luis-Alfonso; Torres-Ochoa, Sergio R.

Evaluación de adquisición de conocimientos de conceptos de ecología en estudiantes de bachillerato tecnológico en México

Revista Iberoamericana de Educación Superior, vol. II, núm. 3, 2011, pp. 130-151

Instituto de Investigaciones sobre la Universidad y la Educación

.jpg, México

Disponible en: http://www.redalyc.org/articulo.oa?id=299124244007

Cómo citar el artículo

- Número completo

- Más información del artículo

Página de la revista en redalyc.org

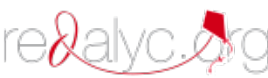

Sistema de Información Científica

Red de Revistas Científicas de América Latina, el Caribe, España y Portugal

Proyecto académico sin fines de lucro, desarrollado bajo la iniciativa de acceso abierto 


\title{
Evaluación de adquisición de conocimientos de conceptos de ecología en estudiantes de bachillerato tecnológico en México
}

\author{
Luis-Alfonso Cuevas-Novoa y Sergio R. Torres-Ochoa
}

\section{Resumen}

Se evaluó la capacidad de asimilación cognitiva de conceptos fundamentales de ecología en dos escuelas de bachillerato tecnológico en el estado de Michoacán, México. Con fundamentos de teoría cognitiva se diseñó y aplicó un instrumento de valoración de cambio cognitivo en una muestra representativa, definida al azar, de estudiantes de ambas escuelas. Los reactivos del instrumento fueron elaborados a manera de proposiciones, con selección de respuesta de doble exclusión a partir de un criterio teórico (definición conceptual). El análisis cuantitativo consistió en valorar estadísticamente el cambio significativo entre antes y después de la intervención docente; el análisis cualitativo identificó relaciones entre conceptos de mapas elaborados por los estudiantes. Los resultados apuntan a una nula, y en la mejor de las situaciones escasa, asimilación cognitiva de conceptos básicos de ecología.

Palabras clave: cognoscitivismo, ecología, educación media superior, educación científica, educación ambiental.

Luis-Alfonso Cuevas-Novoa

cunova@hotmail.com

Maestro en Ciencias por el Centro Interdisciplinario de Investigación y Docencia en Educación Técnica (CIIDET), Secretaría de Educación Pública. Profesor-investigador en la Universidad de la Ciénega, Michoacán, México. Temas de investigación: educación media superior; trayectoria escolar; estudio de curriculum.

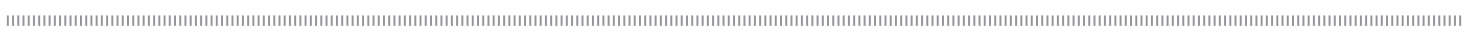




\title{
Avaliação da aquisição de conhecimentos sobre conceitos de ecologia em estu- dantes de ensino médio e técnico em México
}

\section{Resumo}

Avaliou-se a capacidade de assimilação cognitiva de conceitos fundamentais de ecologia em duas escolas de nível médio e técnico no Estado de Michoacán, México. Com fundamento na teoria cognitiva foi desenhado e aplicado um instrumento para avaliar a mudança cognitiva em uma amostra representativa, definida ao azar, de estudantes das duas escolas. Os reagentes do instrumento foram elaborados a maneira de proposições, com seleção de resposta de dupla exclusão a partir de um critério teórico (definição conceitual). A análise quantitativa consistiu em avaliar estatisticamente a mudança significativa entre o antes e o depois da intervenção docente; a análise qualitativa identificou relações entre conceitos de mapas elaborados pelos estudantes. Os resultados apontam a uma nula, e na melhor das situações escassa, assimilação cognitiva dos conceitos básicos da ecologia.

Palavras chave: cognoscitivismo, ecologia, ensino meio superior, educação científica, educação ambiental.

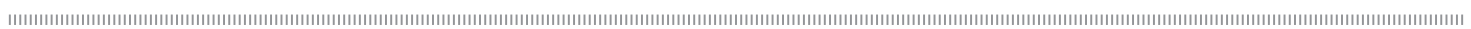

Assessment of knowledge acquisition about ecological concepts in technical high school students in Mexico

\begin{abstract}
The capacity of cognitive assimilation of essential ecological concepts was assessed in two technical high schools in the state of Michoacán, Mexico. A cognitive theory based instrument was designed and administered to assess cognitive change in a randomly defined, representative sample of students from both schools. The reagents of the instrument were prepared as clauses, choosing mutually exclusive answers based on a theoretical criterion (conceptual definition). The quantitative analysis was the statistical valuation of significant change between before and after the educational intervention; the qualitative analysis identified relationships between the concepts of maps prepared by the students. The results point out that the cognitive assimilation of basic ecological concepts is nil or at best limited.
\end{abstract}

Key words: cognitivism, ecology, vocational secondary education, science education, environmental education. 


\section{Introducción}

La realidad hace evidente una deficiente cultura ecológico-ambientalista, de manera genérica, en los países latinoamericanos. En el estado de Michoacán, México, es necesario insistir y revitalizar de manera continua los procesos de enseñanza y aprendizaje escolarizados respecto de este campo de la ciencia y de la sociedad (Torres, 2006: 15). Reorientar las estructuras curriculares hacia la comprensión de la interacción humana con el entorno es una tarea obligada y en permanente revisión. Igualmente, evaluar el impacto de los cambios propuestos en los diferentes niveles de la educación formal es imprescindible para el logro de la formación de ciudadanos comprometidos con la sociedad y el medio ambiente (Torres, 2006).

De acuerdo con Morin (1996) la ecología es la primera ciencia que trata del sistema global integrado por constituyentes físicos, botánicos, sociológicos, microbianos, cada uno de los cuales depende de una disciplina especializada. Es decir, la ecología es una ciencia compleja que trasciende la visión disciplinaria hacia una policompetencia de diferentes dominios con una fuerte tendencia hacia la multi e interdisciplina (Oyama, 2002).

El conocimiento ecológico necesita, sobre todo, una aprehensión de las interacciones y de su naturaleza sistémica (Morin, 1996). Estamos pues en presencia de una ciencia de nuevo tipo, sustentada sobre un sistema complejo, que apela a la vez a las interacciones particulares y al conjunto global que, además, resucita el diálogo y la confrontación entre los hombres y la naturaleza y permite las intervenciones mutuamente provechosas para unos y otra (Morin, 1996).

No se pude entender la transdiciplinaridad de la ciencia ecológica sin entender la naturaleza sistémica y la base epistemológica multidimensional que, como ciencia, le aportan disciplinas como la física y la química, pero en especial la biología (Torres, 2006: 12). Así, la conceptuación ecológica básica tiene especial valor, pues aporta elementos fundamentales para la comprensión de las relaciones de la especie humana con su entorno. Los conceptos son una herramienta fundamental en la formación escolarizada, dadas las nociones científicas que el cognitivismo nos da (Torres, 2006: 2).

El estudio aquí presentado parte de la base de que la ecología es el sustento epistemológico para el entendimiento de cualquier problemática referida a las interrelaciones hombre-naturaleza. Nos ofrece el conocimiento de las múltiples relaciones que se suceden en el planeta entre los organismos que habitan en esta gran casa que compartimos, organizada en poblaciones, comunidades y ecosistemas (Curiel, 2004).

En el marco de la reforma curricular del bachillerato tecnológico en México, la implementación de la materia ecología como nueva asignatura dentro de la estructura básica del plan de estudios no es ajena a esta tendencia (COSNET, 2004).

En este estudio se parte de considerar al estudiante del nivel bachillerato como a un sujeto que es capaz de pensar, actuar y sentir para construir nuevos conocimientos por sí mismo, así como de construir relaciones autónomamente con su entorno. Es un sujeto que, durante la construcción de su propio conocimiento, desarrolla a plenitud las dimensiones intelectual, afectiva y de valores.

El aprendizaje no se puede reducir a un problema exclusivamente pedagógico o técnico; tiene raíces profundas en el significado que el estudiante otorga a la educación, la vida y el entorno (Degua, 2005). Es también un proceso durante el cual el aprendiz recorre un camino y, en ese recorrido, va dando cuenta de sus aprendizajes a partir de diversos productos que puede elaborar en distintos momentos. Uno de ellos es la participación comunicativa entre profesor y alumno: "En ambos casos se establece un proceso cognitivo de construcción analítica compleja: deconstrucción y reconstrucción lógico-conceptual" (Campos y Gaspar, 2004). 
Berger y Luckmann (2001) insisten en el hecho de que la construcción del conocimiento parte de la realidad subjetiva, por lo que no podemos descartar la dimensión cultural en el proceso de aprendizaje, lo que en términos de teoría cognitiva significa que:

la arquitectura intracerebral del control atencional y del apercibimiento representacional logrados por el cerebro humano - como efectos de la culturización sobre la competencia adaptativa individual - han resultado en una conciencia humana radicalmente distinta a la de sus parientes vivientes y a la de sus antecesores (Fernández y Gumá, 2001: 343).

Dentro de esta visión, la educación media superior es considerada como un espacio de grandes oportunidades de construcción de visiones del mundo, en donde los sistemas educativos pueden incidir de mejor manera para rescatar una amplia diversidad de pensamientos e ideas. Esto es, el entorno de culturización sistemática, en nuestro caso, está delimitado al desempeño escolar medio superior. En cuestiones ecológicas el bachillerato es la etapa y el contexto cultural oportunos para incidir en el desarrollo de opciones cognitivas, afectivas y prácticas, más que en ningún otro nivel escolar (Curiel, 2004).

El planteamiento problemático de la investigación aquí reseñada se concentra en los siguientes cuestionamientos:

- ¿La apropiación de conceptos ecológicos en estudiantes del nivel bachillerato es consistente con la intencionalidad plasmada en su plan de estudios?

- ¿Es posible evidenciar estructuras de reconocimiento lógico de conceptos de ecología en estudiantes del bachillerato tecnológico como consecuencia de intervención docente?
La respuesta a estas interrogantes sobre asimilación de conocimiento de conceptos básicos de ecología en el nivel educativo que nos ocupa llevaron a plantearnos el siguiente:

Objetivo general del estudio: valorar el nivel cognitivo sobre aspectos básicos conceptuales de ecología en estudiantes del bachillerato tecnológico.

Nuestro fundamento teórico estriba en que la expresión de las ideas a través del lenguaje (el discurso), en este caso de orden científico, es evidencia de organización mental de esas ideas en un contexto socializante. Esto lo sustenta la afirmación de que:

La creciente encefalización, junto con la lateralidad hemisférica y la plasticidad del cerebro humano tienen alta correlación con su progresiva ‘culturización' y socialización, y con el desarrollo de la comunicación mimético-vocal cuyo resultado fue el lenguaje hablado y escrito [lo anterior, aunado a que] la evolución de los sistemas representacionales intracerebrales en el humano ha relegado a un segundo plano, subordinado, a los componentes cerebrales de los sistemas ejecutivos centrales comunes al diseño básico del cerebro mamífero (Fernández y Gumá, 2001: 342-343).

La base para la utilización de un instrumento diseñado para evaluar cambio cognitivo tuvo que tomar en consideración, a despecho de un diseño curricular de un curso tradicional de ecología, el fundamento teórico de que: "Las tareas cognitivas no son resueltas mediante una progresión jerárquica secuencial hacia el objetivo, sino mediante la consideración simultánea e interactiva de múltiples posibilidades y soluciones, hasta lograr un ajuste satisfactorio" (ibid.: 348).

El modelo utilizado toma en consideración que "el aprendizaje de conocimientos específicos depende por completo del desarrollo de estructuras cognitivas generales, que él formaliza en términos lógicos", de acuerdo con Piaget (citado por Pozo, 
2002: 178), y donde resalta el equilibrio entre procesos de asimilación y acomodación; conceptos que, teóricamente, intentan explicar cómo conocemos el mundo y cómo cambia nuestro conocimiento sobre el mundo. La asimilación deviene en un "proceso por el que el sujeto interpreta la información que proviene del medio, en función de sus esquemas o estructuras conceptuales disponibles" (idem.). De esa forma, para Piaget "la categorización conceptual sería un claro ejemplo de asimilación de un objeto a un esquema cognitivo" (ibid.: 179). El ejemplo que refiere Pozo es: "Aunque los sujetos se ven enfrentados a un estímulo carente de significado, [por ejemplo] una simple y caprichosa mancha de tinta, asimilan ese estímulo a uno de sus esquemas o conceptos disponibles: el concepto de murciélago o de mariposa [según el propio ejemplo]" (Pozo, 2002: 179). Piaget (citado por Pozo, 2002: 180) define acomodación como: "cualquier modificación de un esquema asimilador o de una estructura, modificación causada por los elementos que se asimilan". Es decir, la acomodación supone modificación de esquemas previos —en función de información asimilada - pero también nueva asimilación (reinterpretación) de datos o conocimientos anteriores en función de nuevos esquemas construidos. Se agrega aquí que "sólo de los desequilibrios entre estos dos procesos surge el aprendizaje o el cambio cognitivo" (ibid.: 181), sin perder de vista que "el aprendizaje de los conceptos sólo puede explicarse desde posiciones que establezcan una interacción efectiva entre asociación y reestructuración" (ibid: 191). Lo anterior intenta plasmarse en el diseño del instrumento de evaluación cognitiva aquí referido: asociación sintáctica proposicional y forzar a la reestructuración al proceder a seleccionar la estructura proposicional lógica, tal y como se describe más adelante.

La influencia de Vygotsky aparece en nuestro trabajo cuando consideramos que "los conceptos verdaderos son los conceptos científicos adquiridos a través de la instrucción. A diferencia de los conceptos espontáneos, los conceptos científicos tienen tres rasgos característicos en su adquisición" (citado por Pozo, 2002: 202-203): los conceptos científicos forman parte de un sistema, se adquieren por toma de conciencia de la propia actividad mental e implican relación especial con el objeto por internalización de la esencia del propio concepto.

No puede obviarse el aporte de Ausubel quien, respecto del aprendizaje significativo, señala que el material por aprender no debe ser arbitrario (Campos y Gaspar, 1996: 56) (en nuestro caso, conceptos fundamentales de la ecología, de conformidad con expertos); es importante también la predisposición del sujeto, variable para la cual, en el estudio, no hubo más garantía que confiar en que los ejemplos de especies biológicas endémicas que se utilizaron en el instrumento de evaluación (pez blanco y mariposa monarca) como base teórica, para luego responder los reactivos, presentan rasgos de afinidad cultural por parte de los estudiantes de la muestra.

\section{Metodología}

La referencia básica del esquema conceptual del estudio son seis conceptos considerados como fundamentales (Burns, 1992) - al respecto hay controversia (Scheiner, Hudson y Vandermeulen, 1993), sin embargo, como recurso epistemológico para la definición conceptual de la ecología, resulta altamente significativo - y cuatro conceptos que son reconocidos como complementarios (Torres, 2006: 56). Además, los conceptos adicionales (13) que el programa de educación tecnológica marca como de especial interés educativo (COSNET, 2004), se redistribuyeron dentro de los fundamentales y los complementarios, por correspondencia de inclusión (subordinación semántica).

Respecto de los instrumentos de recogida de datos, se utilizaron técnicas cualitativas y cuantitativas de análisis, coincidiendo con Jiménez 
(1989) en que los dos tipos de técnicas suministran datos complementarios y proporcionan mayor riqueza en un estudio que se ocupa de ambas visiones científicas.

Con base en los criterios metodológicos anteriores, se procedió al diseño de un instrumento de evaluación cognitiva, de examen de ensayo y examen de items (Campos, Cortés y Gaspar, 1999).

El instrumento constó de dos partes (Torres, 2006); la primera, que evalúa la capacidad cognitiva de manera cuantitativa, está estructurada por 25 reactivos de respuesta cerrada, mismos que tienen relación directa o indirecta con los conceptos del esquema de referencia. La segunda parte presenta dos casos de fauna endémica del estado de Michoacán (pez blanco y mariposa monarca), estructurados en términos proposicionales, considerando relaciones lógicas entre conceptos; después de su lectura se hicieron preguntas abiertas con el fin de evaluar la capacidad de asimilación. La fase analítica permitió estructurar mapas conceptuales por cada argumento expresado por los estudiantes de la muestra, y cuya valoración se basó en el esquema propuesto por Novak (Novak y Gowin, 1988).

Diversos autores critican e incluso refutan los modelos de cambio conceptual en la educación en ciencias (De Posada, 2002:101-104); sin embargo, este estudio, relacionado con modelos de cambio conceptual, explicita la posibilidad de su valoración con base en el reconocimiento de estructuras lógicas del discurso científico, sin llegar a planteamientos intervinientes en la docencia, que son mucho más complejos y requieren simultaneidad de factores, más allá de los aspectos propiamente cognitivos. Por ejemplo, una propuesta de intervención integradora señala que: "El conocimiento declarativo que tenemos sobre el mundo es susceptible de ser dividido en elementos de memoria semántica rutinaria, memoria semántica significativa y memoria episódica" (ibid:: 106), lo cual rebasa nuestro problema de investigación. Éste se concentra en aspectos de contenido epistemológico y organización conceptual (Campos, Cortés y Gaspar, 1999; Campos y Cortés, 2005), respecto de su modificación entre antes y después de la intervención docente.

Por otro lado, hay opiniones claras respecto de que: "En lo relativo a los aprendizajes conceptuales, existe consenso en la necesidad de la construcción de modelos interpretativos sobre los principales campos conceptuales de la biología: evolución, nutrición, reproducción, relación, célula, organismo vivo, ecosistema" (Bermudez y de Longhi, 2008: 280), la cual es una ruta cercana a nuestro planteamiento.

\section{Formato de trabajo}

La aplicación del instrumento se realizó como pre-prueba y post-prueba, que coincidieron, respectivamente, con el principio y terminación del curso de ecología en el plan curricular de dos centros de bachillerato tecnológico: Centro de Estudios Tecnológico Industrial y de Servicios (CETIS) 121 y Centro de Bachillerato Tecnológico Industrial y de Servicios (CBTIS) 12, localizados en los municipios de Sahuayo y Jiquilpan, Michoacán. Se tomaron muestras representativas de alumnos del cuarto semestre correspondientes aproximadamente al $10 \%$ de la población estudiantil; la elección se hizo aleatoriamente de la totalidad de grupos de ese semestre: 50 alumnos por escue1a. La consignación de respuestas se estableció de acuerdo con el siguiente criterio: a) 0.5 , para respuestas sin estructura lógica; b) 1 , para las respuestas con estructura lógica; c) 0, para la opción NO RECUERDO. Es decir, cada reactivo (pregunta del instrumento de valoración) se estructuró a manera de proposición incompleta, y el estudiante debía seleccionar entre dos opciones de complementación (una que reúne características de estructuración lógica y otra que no); o bien seleccionar la opción: NO RECUERDO. 
El análisis estadístico se realizó con base en la prueba de distribución de $T$ de Student, para cada reactivo, considerando el promedio de respuestas de los 100 estudiantes (separados por escuela), para establecer el contraste entre la asimilación cognitiva de conceptos de ecología antes y después de la intervención docente (pre-prueba versus post-prueba).

Los mapas conceptuales se diseñaron con base en las respuestas de los tres alumnos con mayor calificación cuantitativa, en promedio, de los 25 reactivos del instrumento de evaluación; así como con los tres estudiantes con menor calificación. Se elaboraron mapas conceptuales de referencia (criterio) con base en los argumentos proposicionales de los dos ejemplos biológicos de la segunda parte del instrumento. Los mapas criterio se contrastaron cualitativamente con los elaborados a partir de las respuestas de los dos grupos de estudiantes señalados.

\section{Base de conceptos abordados en el estudio (instrumento cognitivo) Conceptos fundamentales}

Flujo de energía. Nos permite situarnos en una racionalidad física desde la cual los organismos somos parte de un flujo energético que mantiene en movimiento termodinámico las expresiones externas e internas de vida.

Nicho ecológico. Se refiere a la función que ejerce cada especie dentro del ecosistema y en donde cada una ocupa un espacio concreto dentro del ambiente: desde su formulación se hace referencia al espacio-función. Está relacionado con el eje de la biodiversidad, donde cada especie tiene su nicho ecológico y la desaparición de alguna de ellas acarrea desestabilización para las restantes.

Conservación de recursos. Concepto estrechamente relacionado con la planificación y uso racional de los mismos. Es uno de los conceptos que interpretan mejor al ambiente a través de la identificación de amenazas generadas por nuestros estilos de vida.
Competencia. Resulta cuando varios individuos de la misma especie o de varias especies dependen del mismo recurso limitado. La competencia entre individuos de distintas especies (competencia interespecífica) es una de las principales materias de estudio de la ecología. La competencia entre individuos de la misma especie (competencia intraespecífica) es uno de los principales mecanismos de selección natural.

Sucesión. Es la secuencia o cambio de organismos con el paso del tiempo en una comunidad o hábitat.

Ecosistema. Designa a todo el sistema de organismos asociados, junto con los factores físicos de su entorno.

\section{Conceptos complementarios}

Distribución. Es el número de individuos o grupo de especies que ocupan un territorio especifico y que está controlado por los requisitos del nicho de la especie.

Organismo. Todo lo que cumpla con las características esenciales para considerarlo vivo desde una perspectiva biológica (teoría celular).

Hábitat. Entendido como el lugar donde vive y se desarrolla un organismo.

Ambiente. Es la atmósfera que rodea un cuerpo; conjunto de condiciones tanto físicas como químicas.

\section{Conceptos curriculares adicionales}

Éstos se redistribuyen semánticamente entre los 6 fundamentales y los 4 complementarios del modelo cognitivo utilizado (Torres, 2006): Factores bióticos y abióticos, Cadena trófica, Equilibrio, Productores, Consumidores, Recursos naturales, Comunidad, Poblaciones, Contaminación, Sustentabilidad, Biosfera, Biodiversidad, Bioma.

En la tabla 1 se distribuye por correspondencia técnica a los conceptos fundamentales y complementarios con los reactivos del instrumento. 
Tabla 1. Base conceptual correspondiente a los reactivos del instrumento y conceptos curriculares relacionados

\begin{tabular}{|c|c|c|}
\hline Concepto & Reactivo & Concepto curricular relacionado \\
\hline A. Sucesión & 3,13 & Sucesión \\
\hline B. Flujo de energía & $\begin{array}{c}11,14,20,15 \\
16\end{array}$ & $\begin{array}{l}\text { Flujo de energía, Cadena trófica, Equilibrio, } \\
\text { Productores, Consumidores. }\end{array}$ \\
\hline $\begin{array}{l}\text { C. Conservación de } \\
\text { recursos }\end{array}$ & $3,4,25$ & $\begin{array}{l}\text { Recursos naturales, Conservación de } \\
\text { recursos, Sustentabilidad }\end{array}$ \\
\hline D. Competencia & 17,18 & Competencia interespecífica e intraespecífica \\
\hline E. Nicho & 5,12 & Nicho, Biodiversidad \\
\hline F. Ecosistema & $1,7,9,10$ & Ecosistema, Factores bióticos y abióticos \\
\hline G. Distribución & 6,23 & Comunidad, Poblaciones \\
\hline H. Organismo & 22 & Organismo \\
\hline I. Hábitat & 8,21 & Biosfera, Hábitat \\
\hline J. Ambiente & 2,19 & Medio ambiente, Contaminación \\
\hline
\end{tabular}

Tabla 2. Niveles cognitivos utilizados y su correspondencia con los reactivos del instrumento y con los conceptos (fundamentales y complementarios)

\begin{tabular}{|c|c|c|}
\hline Nivel cognitivo & Reactivo & Concepto relacionado \\
\hline Explicativo & $\begin{array}{l}1,7,11,13,20 \\
22,23,24,25\end{array}$ & $\begin{array}{l}\text { Ecosistema, Flujo de energía, Sucesión, } \\
\text { Hábitat, Organismo, Distribución. }\end{array}$ \\
\hline Descriptivo & $\begin{array}{c}2,3,5,12 \\
14,17,18,19\end{array}$ & $\begin{array}{l}\text { Ambiente, Conservación de recursos, } \\
\text { Nicho, Competencia. }\end{array}$ \\
\hline Ejemplificativo & $\begin{array}{c}4,6,8,9 \\
10,15,16,23\end{array}$ & Conservación de recursos. \\
\hline
\end{tabular}


Cada uno de los 25 reactivos del instrumento de evaluación cognitiva se corresponden con alguno de los niveles cognitivos asumidos en el estudio (Campos y Cortés, 2005): explicativo, descriptivo, ejemplificativo. Igualmente, cada reactivo está relacionado con alguno de los conceptos a evaluar. Lo anterior se expresa en la tabla 2.

\section{Análisis de resultados cuantitativos: conceptos con diferencia significativa}

Se encontró diferencia significativa, entre preprueba y post-prueba, en cuatro conceptos para el CETIS 121 y en tres para el CBTIS 12 (cambio cognitivo detectado al comparar resultados entre ambas pruebas).

En el caso del CETIS 121, los conceptos con significancia estadística (diferencia significativa entre pre-prueba y post-prueba) fueron: ecología, factores bióticos, biodiversidad, organismo.

El reactivo número 1 se utilizó como base para valorar el concepto general de ecología; se categorizó en el nivel cognitivo explicativo y relacionado epistemológicamente con el concepto fundamental ecosistema. Es indispensable para favorecer la comprensión de la interdependencia de los seres vivos y el medio, así como para marcar la diferencia con la biología. La cabal dimensión del concepto permite al estudiante un punto de partida fundamental para el análisis de la realidad ambiental: presentó una dinámica muy particular; desde la pre-prueba presentó un alto valor de significatividad (y por esa razón también aparece en la tabla 3) y se incrementó en la post-prueba. Hay claridad en la comprensión del concepto desde el inicio del estudio. Por otra parte, la diferencia significativa entre pre-prueba y post-prueba enfatiza la intervención docente como un factor favorable en la modificación del aprendizaje de este concepto en el conjunto de alumnos de la muestra.
El reactivo factores bióticos se categorizó en el nivel cognitivo ejemplificativo, relacionado epistemológicamente con el concepto fundamental ecosistema. Epistemológicamente es imprescindible para cualquier tratamiento esquemático conceptual de ecología. Cognitivamente, por su carácter ejemplificativo, está sujeto a relaciones obligadas con conceptos explicativos. Entender que todos los organismos tienen cierto nivel de interacción que afecta su desempeño así como su abundancia y distribución permitirá modificar las concepciones de los estudiantes sobre ambiente en general y el componente biótico en particular.

Biodiversidad se categorizó en el nivel cognitivo descriptivo, relacionado epistemológicamente con el concepto fundamental de nicho ecológico. La biodiversidad se entiende aquí como la tipología y cantidad de especies diferentes que ocupan un lugar determinado; requiere para su cabal entendimiento de la identificación de la relación con conceptos como factores bióticos y abióticos. Es importante recalcar que el concepto de nicho, con el cual se le relaciona aquí, es uno de los que presentó mayor dificultad de asimilación.

Organismo es un reactivo categorizado como explicativo. Es prioritario para la comprensión de las relaciones ecológicas cuyo desconocimiento impediría o dificultaría enormemente el aprendizaje de aspectos organizativos: competencia, sucesión y flujo de energía, los cuales, a su vez, están asociados a una serie de principios básicos de ecología: competencia intra e interespecífica, así como la segunda ley de la termodinámica (flujo de energía).

En el caso del CBTIS 12, los conceptos que presentaron diferencia estadística significativa fueron: conservación de recursos, nicho, biosfera.

Conservación de recursos se categorizó en el nivel cognitivo descriptivo. Epistemológicamente se relacionó con el concepto fundamental conservación de recursos. Toda especie tiene la necesidad 
de obtener satisfactores que le permitan subsistir y perpetuarse, por lo cual, el conocimiento de conservación de recursos permite la visualización de la modificación del ecosistema y, por ende, el grado de conservación de aquellos. Se sitúa en el contexto de reconocer al ser humano como una especie más de la trama ecológica, relacionando sus actividades con el resto de las especies. Aun cuando hay cambio estadístico significativo entre pre-prueba y post-prueba, en esta última no alcanza, en promedio, a superar el valor crítico que evidencia cambio cognitivo (0.5), y por esta razón también aparece en la tabla 4 . Se tomó el criterio de admitir el cambio estadístico a reserva de que no cumple con el valor crítico aceptado.

Nicho ecológico es categorizado en el nivel cognitivo descriptivo. Su asimilación conceptual repercute en el entendimiento de las relaciones entre los organismos en los ecosistemas, así como los aspectos filogenéticos. El nicho ecológico resume los factores ambientales que influyen en el crecimiento, supervivencia y reproducción de una especie en particular; se relaciona directamente con los conceptos distribución y poblaciones. En el estudio presentó los niveles estadísticos de significancia más bajos (en concordancia con bajos niveles de modificación cognitiva, comparativamente entre pre-prueba y post-prueba). En la post-prueba no alcanzó el valor 0.5 - establecido aquí como valor crítico para ser considerado con significancia cognitiva-, por lo cual se puede afirmar que no se detectó cambio significativo en el aprendizaje.

Biosfera fue categorizado en el nivel cognitivo ejemplificativo, relacionado epistemológicamente con el concepto complementario hábitat. Jerárquicamente corresponde al nivel más alto dentro de los niveles de organización de la materia en el sistema viviente, que se incluye dentro del ámbito de la ecología global.

Este concepto se relaciona con el lugar donde viven los seres vivos, se plantea desde cursos anteriores de educación básica en el área de biología y geografía, pero en no pocas ocasiones se limita sólo al entendimiento de la dimensión espacial, sin tomar en cuenta el total de interacciones de las especies de la biosfera con el medio. Este concepto en el estudio mostró diferencia significativa entre pre-prueba y post-prueba. Es decir, se constata cambio cognitivo: arriba del valor crítico estadístico de 0.5 en el conjunto de la muestra como promedio, en la post-prueba.

\section{Análisis de los conceptos sin diferencia significativa}

Hubo algunos conceptos que, desde el inicio del estudio (pre-prueba), evidenciaron asimilación conceptual con base en el criterio de reconocimiento de estructuras lógicas, que es el criterio que utiliza el instrumento de valoración cognitiva. Se trata de conocimientos previos con suficiencia científica y por tanto no relacionados con la intervención docente.

El análisis de los resultados pone de manifiesto la comprensión previa (cuadros sombreados de tabla 3), por parte de los estudiantes de la muestra, de ciertos conceptos, lo cual significa evidencia inicial de asimilación de los mismos (conocimiento previo). Para la muestra del CETIS 121: ecología, medio ambiente, consumidores, contaminación, equilibrio ecológico, hábitat y distribución de poblaciones. Para CBTIS 12: ecología, medio ambiente, comunidad, factores abióticos, factores bióticos, biodiversidad, consumidores, contaminación, equilibrio ecológico, hábitat, organismo y distribución de poblaciones. Hay coincidencia en ambas escuelas para: ecología, ambiente, consumidores, contaminación, equilibrio ecológico, hábitat, distribución de poblaciones.

Ambiente es un concepto que hace referencia al entorno que afecta y condiciona especialmente las circunstancias de vida, y no sólo el espacio en el que se desarrolla ésta. Requiere del dominio de conceptos como factores bióticos y abióticos. 
Tabla 3. Conceptos con evidencia de asimilación previa al curso de ecología

\begin{tabular}{|c|c|c|}
\hline Concepto & CETIS 121 & CBTIS 12 \\
\hline \multicolumn{3}{|c|}{ 1. Ecología (ecosistema) } \\
\hline \multicolumn{3}{|l|}{ 2. Ambiente } \\
\hline \multicolumn{3}{|l|}{ 3. Recursos naturales } \\
\hline \multicolumn{3}{|c|}{ 4. Conservación de recursos } \\
\hline \multicolumn{3}{|l|}{ 5. Nicho } \\
\hline \multicolumn{3}{|l|}{ 6. Comunidad } \\
\hline \multicolumn{3}{|l|}{ 7. Ecosistema } \\
\hline \multicolumn{3}{|l|}{ 8. Biosfera } \\
\hline \multicolumn{3}{|l|}{ 9. Factores bióticos } \\
\hline \multicolumn{3}{|l|}{ 10. Factores abióticos } \\
\hline \multicolumn{3}{|l|}{ 11. Flujo de energía } \\
\hline \multicolumn{3}{|l|}{ 12. Biodiversidad } \\
\hline \multicolumn{3}{|l|}{ 13. Sucesión } \\
\hline \multicolumn{3}{|l|}{ 14. Cadena trófica } \\
\hline \multicolumn{3}{|l|}{ 15. Productores } \\
\hline \multicolumn{3}{|l|}{ 16. Consumidores } \\
\hline \multicolumn{3}{|c|}{ 17. Competencia interespecífica } \\
\hline \multicolumn{3}{|c|}{ 18. Competencia intraespecífica } \\
\hline \multicolumn{3}{|l|}{ 19. Contaminación } \\
\hline \multicolumn{3}{|c|}{ 20. Equilibrio ecológico } \\
\hline \multicolumn{3}{|l|}{ 21. Hábitat } \\
\hline \multicolumn{3}{|l|}{ 22. Organismo } \\
\hline \multicolumn{3}{|c|}{ 23. Distribución de poblaciones } \\
\hline \multicolumn{3}{|l|}{ 24. Bioma } \\
\hline 25. Sustentabilidad & & \\
\hline
\end{tabular}


El concepto consumidores está relacionado con el de flujo de energía en los ecosistemas.

El concepto contaminación, entendido como factor ambiental, físico, químico o biológico que por encima de cierto nivel causa daño a los organismos, es un concepto ampliamente difundido por lo que el grado de comprensión del concepto no presenta mayor dificultad.

El concepto equilibrio ecológico está estrechamente relacionado con el concepto fundamental de flujo de energía, pero la percepción general se encamina más al estado de balance de los componentes del ecosistema integrados al concepto ambiente. Se identifica por las amenazas generadas por nuestros estilos de vida y la relación de inconsciencia para con la naturaleza, que nos hace vulnerables a las diversas circunstancias generadas por las continuas alteraciones al orden del medio (Leff, 2000).

Hábitat es uno de los conceptos que implica el menor grado de dificultad, posiblemente debido a su relación directa con el espacio físico del desarrollo de los organismos. Es un concepto sencillo y claro, fácil de entender e interpretar y no presenta mayor ambigüedad.

En el caso de la distribución de poblaciones desde la perspectiva ecológica, es necesaria la comprensión de los factores ambientales, bióticos y abióticos como parte trascendente para la comprensión de los patrones de distribución de las poblaciones.

$\mathrm{E}$ l hecho de que los estudiantes de ambas escuelas muestren asimilación previa de este concepto en particular, manifiesta un grado básico suficiente de articulación para el manejo del tratamiento esquemático ecológico.

\section{Análisis de conceptos con nula - o no detectable - asimilación conceptual}

Los conceptos que presentaron nula asimilación conceptual (sin cambio significativo entre pre-prueba y post-prueba) se muestran en la tabla 4 (cuadros sombreados). La intervención docente es insuficiente o nula, $15(60 \%)$ para CETIS 121 y 11 (44\%) para CBTIS 12.

\section{Análisis de resultados cualitativos: mapas conceptuales}

La valoración del mapa conceptual se hizo atendiendo al esquema propuesto por Novak y Gowin (1988), acondicionada al propósito de la investigación de acuerdo con Ontoria et al. (2000), quienes sugieren que cada profesor puede hacer su escala propia, a nivel personal.

Para ello es importante tomar en cuenta los cuatro criterios básicos para la evaluación de mapas conceptuales: las proposiciones, la organización jerárquica de la estructura cognoscitiva, las relaciones cruzadas y los ejemplos, que constituyen los parámetros para la valoración de un mapa conceptual:

- Las proposiciones, es decir, los conceptos con las palabras-enlace apropiadas, que indican relaciones válidas o erróneas.

- La jerarquización o estructura jerárquica del mapa, en el sentido de que los conceptos más generales incluyan a los más específicos y éstos a su vez estén dibujados debajo de uno más general.

- Las relaciones cruzadas, que muestran relaciones entre conceptos pertenecientes a partes diferentes del mapa conceptual. Las conexiones significativas y válidas entre los distintos segmentos de la jerarquía conceptual.

- Los ejemplos, en ciertos casos, para estar seguros de que los alumnos han sabido comprender la expectativa de lo que es concepto y lo que no lo es: acontecimientos y objetos concretos válidos de lo que designa el concepto. Es menester recordar que los ejemplos no van encerrados en un círculo ya que no constituyen conceptos. 
Tabla 4. Conceptos con nula asimilación conceptual comparada entre pre-prueba y post-prueba (sombreados)

\begin{tabular}{|c|c|c|}
\hline Concepto & CETIS 121 & CBTIS 12 \\
\hline \multicolumn{3}{|l|}{ 1. Ecología } \\
\hline \multicolumn{3}{|l|}{ 2. Medio ambiente } \\
\hline \multicolumn{3}{|c|}{ 3. Recursos naturales } \\
\hline \multicolumn{3}{|c|}{ 4. Conservación de recursos } \\
\hline \multicolumn{3}{|l|}{ 5. Nicho } \\
\hline \multicolumn{3}{|l|}{ 6. Comunidad } \\
\hline \multicolumn{3}{|l|}{ 7. Ecosistema } \\
\hline \multicolumn{3}{|l|}{ 8. Biosfera } \\
\hline \multicolumn{3}{|l|}{ 9. Factores bióticos } \\
\hline \multicolumn{3}{|c|}{ 10. Factores abióticos } \\
\hline \multicolumn{3}{|l|}{ 11. Flujo de energía } \\
\hline \multicolumn{3}{|l|}{ 12. Biodiversidad } \\
\hline \multicolumn{3}{|l|}{ 13. Sucesión } \\
\hline \multicolumn{3}{|l|}{ 14. Cadena trófica } \\
\hline \multicolumn{3}{|l|}{ 15. Productores } \\
\hline \multicolumn{3}{|l|}{ 16. Consumidores } \\
\hline \multicolumn{3}{|c|}{ 17. Competencia interespecífica } \\
\hline \multicolumn{3}{|c|}{ 18. Competencia intraespecífica } \\
\hline \multicolumn{3}{|l|}{ 19. Contaminación } \\
\hline \multicolumn{3}{|c|}{ 20. Equilibrio ecológico } \\
\hline \multicolumn{3}{|l|}{ 21. Hábitat } \\
\hline \multicolumn{3}{|l|}{ 22. Organismo } \\
\hline \multicolumn{3}{|c|}{ 23. Distribución de poblaciones } \\
\hline \multicolumn{3}{|l|}{ 24. Bioma } \\
\hline 25. Sustentabilidad & & \\
\hline
\end{tabular}


Dentro del enfoque cognitivo, el aprendizaje es sobre todo un proceso interno y no sólo basado en comportamientos observables, por lo que la valoración de mapas conceptuales con organización proposicional, en relación con los conceptos propuestos en el instrumento de evaluación, nos permite valorar el grado de significatividad de los conceptos ecológicos asimilados por los alumnos que conformaron la muestra de estudio.

Se seleccionaron tres alumnos que mostraron la mayor calificación en aplicación de instrumento cuantitativo y tres con menor calificación, tanto en pre-prueba como en post-prueba, en las dos escuelas objeto del estudio. De la sección de preguntas abiertas del mismo instrumento, sobre dos especies de fauna endémica (pez blanco y mariposa monarca), se construyeron mapas conceptuales.

Para analizar los mapas conceptuales que elaboraron los estudiantes, se asignaron valores del $50 \%$ al criterio de proposiciones y $50 \%$ al de jerarquía; estos porcentajes se distribuyeron con base en el número de mapas por alumno.

Los mapas conceptuales resultantes se compararon con los mapas criterio como base para el análisis.

\section{Análisis de mapas conceptuales para el CETIS 121}

Los mapas resultantes de los estudiantes del CETIS presentaron la distribución entre pre-prueba y post-prueba como se muestra en la tabla 5.

\section{Conceptos específicos en alumnos del CETIS 121 con menor y mayor calificación en la pre-prueba}

De los alumnos de la primera categoría (menor calificación: 17, 26 y 4), respecto a la pregunta sobre el concepto de distribución, el alumno 17 no evidenció relación conceptual; los alumnos 26 y 4 relacionaron pertinentemente los conceptos para las dos especies involucradas.
Con relación al hábitat del pez blanco, ninguno de los 3 alumnos presentó evidencia de aplicación del concepto.

En el caso del hábitat de la mariposa monarca, el alumno 4 identificó el bioma correspondiente al hábitat y presentó relaciones válidas para el concepto; los dos restantes $(17,26)$ no mostraron relaciones lógicas ni estructura jerárquica. Con respecto a las características más sobresalientes del lepidóptero, los estudiantes 17 y 4 relacionaron bien los hábitos e identificaron correctamente los pedimentos de la pregunta. El estudiante 26 no presentó secuencia lógica alguna. Con respecto a las actividades que ponen en riesgo a la mariposa monarca, los estudiantes 4 y 26 presentaron relación lógica en cuanto a la interacción de los conceptos, así como la utilización de conectores; el estudiante 17 no mostró relación lógica.

De los alumnos con mayor calificación en preprueba (10, 46 y 12), los 3 mostraron argumentación lógica, pero solamente uno utilizó palabras de enlace apropiadas (10), en relación con la distribución del pez blanco; en cuanto al hábitat de este mismo organismo, los tres estudiantes relacionaron adecuadamente el concepto, proposicional y jerárquicamente.

En el caso del hábitat de la mariposa monarca, el estudiante 10 presentó una secuencia jerárquica adecuada y manifestó noción del concepto, pero no un claro dominio del mismo; el estudiante 46 presentó el mismo patrón que el anterior; el mapa del estudiante 12 mostró mayor claridad en la relación de los conceptos, pero sin orden jerárquico. En cuanto a la característica principal del lepidóptero, el alumno 10 mostró adecuada relación de los conceptos, aunque con falta de claridad en la aplicación, con base en el contexto de la pregunta; los estudiantes 46 y 12 fueron claros al reconocer la característica principal de este organismo y las actividades humanas que le ponen en riesgo; en los tres casos hubo claridad en el concepto. 
Tabla 5. Comparación de mapas conceptuales entre pre-prueba y post-prueba del CETIS 121

\begin{tabular}{|c|c|c|c|c|}
\hline $\begin{array}{c}\text { Relación de } \\
\text { alumnos (clave) }\end{array}$ & Proposiciones & Jerarquización & $\begin{array}{c}\text { Relaciones } \\
\text { cruzadas }\end{array}$ & Ejemplos \\
\hline \multicolumn{5}{|c|}{ Alumnos con menor calificación en la pre-prueba } \\
\hline 17CETIS-pre & $10 \%$ & $10 \%$ & - & - \\
\hline 26 CETIS-pre & $20 \%$ & $20 \%$ & - & - \\
\hline 4CETIS-pre & $40 \%$ & $40 \%$ & - & - \\
\hline \multicolumn{5}{|c|}{ Alumnos con mayor calificación en la pre-prueba } \\
\hline 10 CETIS-pre & $40 \%$ & $50 \%$ & - & - \\
\hline 46 CETIS-pre & $45 \%$ & $50 \%$ & - & - \\
\hline 12 CETIS-pre & $50 \%$ & $45 \%$ & - & - \\
\hline \multicolumn{5}{|c|}{ Alumnos con menor calificación en la post-prueba } \\
\hline 26 CETIS-post & $25 \%$ & $25 \%$ & - & - \\
\hline 36 CETIS-post & $40 \%$ & $40 \%$ & - & - \\
\hline 44 CETIS-post & $40 \%$ & $40 \%$ & - & - \\
\hline \multicolumn{5}{|c|}{ Alumnos con mayor calificación en la post-prueba } \\
\hline 2 CETIS-post & $50 \%$ & $50 \%$ & - & - \\
\hline 3 CETIS-post & $50 \%$ & $50 \%$ & - & - \\
\hline 5 CETIS-post & $15 \%$ & $15 \%$ & - & - \\
\hline
\end{tabular}




\section{Alumnos del CETIS 121 con menor y mayor calificación en la post-prueba}

De los estudiantes 26,36 y 44 de la primera categoría (menor calificación), el primero de ellos mostró nula representación proposicional para la distribución del pez blanco. En relación al hábitat de este organismo, manifestó buen nivel en cuanto a proposiciones y jerarquía y ocurrió lo mismo con la mariposa monarca. En el caso de la característica principal de ésta, no manifestó asimilación conceptual en lo que respecta a las actividades que ponen en riesgo al lepidóptero; se observó confusión en la jerarquización de conceptos con respecto a su hábitat.

El alumno 36 presentó conceptuación errónea de la distribución del pez blanco, caso contrario con la identificación de su hábitat, en la cual mostró coherencia lógica. En cuanto al hábitat de la mariposa monarca, identificó claramente el hábitat, proposicional y jerárquicamente; identificó correctamente la característica principal del organismo, así como las actividades que lo ponen en riesgo.

El estudiante 44 presentó para casi todas la preguntas un esquema conceptual pertinente: manifestó relación entre los conceptos y utilizó conectores, con excepción del mapa correspondiente a características de la mariposa, el cual no desarrolló.

En la segunda categoría (con mayor calificación: 2, 3 y 5), el estudiante 2 presentó esquematización pertinente en el conjunto de respuestas, de acuerdo con los criterios propuestos. El alumno 3 presentó el mismo patrón que el anterior, tanto para el criterio proposicional como para el jerárquico.

El estudiante 5 no mostró relaciones lógicas para el hábitat y distribución del pez blanco. Con respecto a preguntas relacionadas con la mariposa, identificó el hábitat pero lo conectó erróneamente; no identificó, al menos esquemáticamente, la característica principal de la mariposa; identificó las actividades de riesgo pero no utilizó los conectores apropiados.

\section{Análisis de mapas conceptuales para el CBTIS 12}

Los mapas de los estudiantes del CBTIS 12 presentaron la distribución que se muestra en la tabla 6 .

\section{Alumnos del CBTIS 12 con menor y mayor calificación en la pre-prueba}

De la primera categoría (con menor calificación: 23,24 y 8 ), el 24 y el 23 relacionaron adecuadamente el concepto de distribución del pez blanco; el estudiante 8 no presentó relación alguna para este concepto. En cuanto al hábitat, ninguno de los tres mostró relaciones pertinentes.

En el caso de la mariposa monarca, los estudiantes 8 y 23 no presentaron indicio de comprensión del concepto y, en todo caso, ideas erróneas respecto de su hábitat; el estudiante 24 presentó mayor claridad en la relación del concepto. En relación con la principal característica del lepidóptero, ninguno de los tres mostró claridad en el concepto. El mismo patrón se observó en el esquema referente a las actividades que ponen en riesgo al organismo.

Los alumnos de la segunda categoría (mayor calificación en la pre-prueba) fueron 3, 21 y 16 . En cuanto a la distribución del pez blanco, el estudiante 16 manifestó concepción errónea; 3 y 21 relacionaron adecuadamente el concepto, con el inconveniente de la falta de conectores para los conceptos. En el caso del hábitat del pez blanco, solamente el 21 presentó relaciones lógicas; el 3 no dio respuesta alguna y el 16 mostró confusión en cuanto a la conceptuación del hábitat.

En cuanto a la mariposa monarca, el estudiante 3 manifestó manejo adecuado del concepto de hábitat, pero sin orden jerárquico; el estudiante 21 mostró buena estructura jerárquica y dominio del concepto; el mismo patrón se observó en el 16 respecto de la característica de la mariposa. Los tres casos mostraron nula relación lógica entre conceptos. En cuanto a las actividades que ponen en riesgo 
Tabla 6. Comparación de mapas conceptuales entre pre-prueba y post-prueba del CBTIS 12

\begin{tabular}{|c|c|c|c|c|}
\hline $\begin{array}{c}\text { Relación de } \\
\text { alumnos (clave) }\end{array}$ & Preposiciones & Jerarquización & $\begin{array}{c}\text { Relaciones } \\
\text { cruzadas }\end{array}$ & Ejemplos \\
\hline \multicolumn{5}{|c|}{ Alumnos con menor calificación en la pre-prueba } \\
\hline 23CBTIS-pre & $10 \%$ & $10 \%$ & - & - \\
\hline 24CBTIS-pre & $20 \%$ & $20 \%$ & - & - \\
\hline 8CBTIS-pre & $0 \%$ & $0 \%$ & - & - \\
\hline \multicolumn{5}{|c|}{ Alumnos con mayor calificación en la pre-prueba } \\
\hline ЗсвTIS-pre & $35 \%$ & $30 \%$ & - & - \\
\hline 21CBTIS-pre & $40 \%$ & $40 \%$ & - & - \\
\hline 16CBTIS-pre & $20 \%$ & $20 \%$ & - & - \\
\hline \multicolumn{5}{|c|}{ Alumnos con menor calificación en la post-prueba } \\
\hline 10CBTIS-post & $35 \%$ & $35 \%$ & - & - \\
\hline 23cBTIS-post & $20 \%$ & $20 \%$ & - & - \\
\hline 34CBTIS-post & $40 \%$ & $40 \%$ & - & - \\
\hline \multicolumn{5}{|c|}{ Alumnos con mayor calificación en la post-prueba } \\
\hline 45CBTIS-post & $30 \%$ & $30 \%$ & - & - \\
\hline 47CBTIS-post & $50 \%$ & $50 \%$ & - & - \\
\hline 50cBTIS-post & $50 \%$ & $50 \%$ & - & - \\
\hline
\end{tabular}


a la mariposa los tres relacionaron adecuadamente los conceptos conectados y jerarquizados.

\section{Alumnos del CBTIS 12 con menor y mayor calificación en la post-prueba}

De la primera categoría (menor calificación: 10, 23 y 34), los mapas correspondientes al estudiante 10 no mostraron relación entre conceptos, así como ninguna jerarquía; presentó concepciones erróneas, excepto en el mapa correspondiente a las actividades que ponen en riesgo a la mariposa.

El estudiante 23 relacionó los conceptos con respecto a la distribución, pero no así para el hábitat, en el caso del pez blanco. En cuanto al hábitat de la mariposa monarca, no mostró ninguna relación conceptual, así como en lo concerniente a las actividades que le ponen en riesgo; en cuanto a sus características, presentó relación adecuada de los conceptos.

El alumno 34 solamente no mostró relación adecuada de los conceptos referentes al hábitat del pez blanco, esto es, sí lo hizo para el resto de los mapas.

De la segunda categoría (mayor calificación: 45,47 y 50) el alumno 45 relacionó adecuadamente los conceptos de distribución para el pez blanco, pero no en caso del hábitat. En las preguntas relacionadas con la mariposa monarca existe confusión en cuanto al hábitat; respecto de las características, así como en lo relativo a las actividades que ponen en riesgo al organismo, presentó claridad conceptual.

El estudiante 47 presentó clara relación de los conceptos, así como orden jerárquico; el estudiante 50 presentó el mismo patrón que el anterior.

\section{Discusión de momento cuantitativo}

De conformidad con los resultados arrojados en la aplicación del instrumento, comparativamente entre ambas escuelas, es factible deducir que hay un mayor manejo conceptual, de manera general, por parte de los alumnos del CBTIS 12; sin embargo, los conceptos biodiversidad, factores bióticos y organismos, presentaron evidencia estadística significativa de modificación cognitiva en el análisis final por parte de los alumnos del CETIS.

La valoración conceptual de los reactivos con nula asimilación prácticamente coincide en las muestras de ambas escuelas. Los conceptos que marcan la diferencia son conservación de recursos $y$ biosfera, pues son los conceptos que, para el caso del CBTIS 12, presentaron evidencia estadística significativa de asimilación conceptual entre preprueba y post-prueba; es decir, no presentaban dominio previo de estos conceptos, en promedio, y lo adquirieron por medio del tratamiento curricular (intervención docente). El concepto nicho ecológico no rebasó el límite crítico de valoración cognitiva, que se fijó en 0.5 (Campos, Cortés y Gaspar, 1999), para considerar que ocurrió un cambio significativo en el aprendizaje del concepto, aun cuando estadísticamente se observó cambio significativo.

Presumiblemente, el impacto del nivel escolar previo con respecto al manejo de conceptos ecológicos que evidenciaron conocimiento previo, no es un factor suficiente para explicar este resultado; un trabajo anterior señala (Torres, 2006: 153) que no existe evidencia sobre asimilación significativa de conceptos ecológicos en la educación secundaria en el estado de Michoacán.

El hecho de que, en promedio, sólo tres conceptos presenten evidencia significativa de asimilación cognitiva entre pre-prueba y post-prueba, puede explicarse por intervención docente. Hay que reconocer que esta evidencia es mínima, ya que no manifiesta un cambio conceptual de mayor alcance: cubre solamente dos conceptos fundamentales $(33 \%)$ de los seis aquí establecidos (el concepto biosfera apenas es un subsidiario de hábitat, concepto complementario).

Por otra parte, no se descarta que el tratamiento epistemológico inadecuado por parte de los docentes incida en esta situación, ya que se ha 
detectado recurrentemente la falta de integración de las ideas científicas en el contexto de la vida cotidiana en la intervención docente (Cortés, 2005), en el tratamiento didáctico (Degua, 2005) y en la gradación de conceptos (Alucema, 2001).

En la tabla 7 se concentran los reactivos del instrumento y los conceptos relacionados, en razón de niveles cognitivos aquí considerados:

\section{Discusión de los resultados cualitativos}

La valoración de los mapas conceptuales manifiesta en general la falta de comprensión de la aplicación de conceptos en contextos determinados; esto a pesar de la evidente claridad en el manejo de algunos conceptos expresados en la parte cuantitativa del estudio. La falta de integración de conceptos ecológicos básicos, aunada a la deficiencia detectada en la expresión esquemática de éstos, así como la incapacidad recurrente para relacionar conceptos, son algunos aspectos que se manifiestan en los esquemas conceptuales, producto de las interrogantes diseñadas en el estudio.

Lo anterior confirma, como señalan Barraza y Cuarón (2004), el aislamiento y fragmentación del conocimiento ambiental, así como la incapacidad de vincular los conceptos con su aplicabilidad, por parte de los jóvenes estudiantes.

Los mapas referidos presentan un carácter idiosincrático (Novak y Gowin, 1988), de acuerdo con la forma en que plasman esquemáticamente el significado de los conceptos los alumnos estudiados. Lo anterior en términos de la experiencia acumulada sobre la realidad que los alude (véase ejemplo de mapa esquema 1) del estudiante $16 \mathrm{del}$ CETIS en aplicación de post-prueba.

\section{Discusión con base en el nivel epistemológico y organización conceptual}

Epistemológicamente, los conceptos cuya asimilación es previa al estudio presentan una distribución categorial más o menos homogénea entre los niveles descriptivo y explicativo, no así para el nivel ejemplificativo. Se observa falta de aplicación de los conceptos en las diferentes circunstancias que plantea el instrumento, como puede verse en el esquema 2.

\section{Tabla 7. Correlación entre nivel cognitivo, reactivos y conceptos}

\begin{tabular}{|c|c|c|}
\hline Nivel cognitivo & Reactivo & Concepto relacionado \\
\hline Explicativo & $\begin{array}{c}1,7,11,13,20, \\
22,23,24,25\end{array}$ & $\begin{array}{c}\text { Ecología, Ecosistema, Flujo de Energía, } \\
\text { Sucesión, Equilibrio, Hábitat, Organismo, } \\
\text { Distribución, Sustentabilidad. }\end{array}$ \\
\hline Descriptivo & $\begin{array}{c}2,3,5,12, \\
14,17,18,19\end{array}$ & $\begin{array}{c}\text { Medio ambiente, Recursos naturales, Nicho, } \\
\text { Biodiversidad, Competencia, Contaminación }\end{array}$ \\
\hline Ejemplificativo & $\begin{array}{c}4,6,8,9, \\
10,15,16,23\end{array}$ & $\begin{array}{c}\text { Conservación de recursos, Comunidad, } \\
\text { Biosfera, Factores bióticos y abióticos, Bioma, } \\
\text { Productores, Consumidores }\end{array}$ \\
\hline
\end{tabular}




\section{Esquema 1. 16 CETIS post (-)}

Distribución del pez blanco

istribución del pez blanco

Distribución restringida

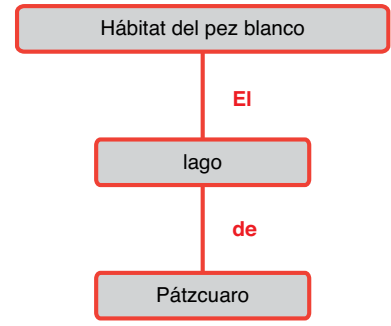

Hábitat de la mariposa monarca En los
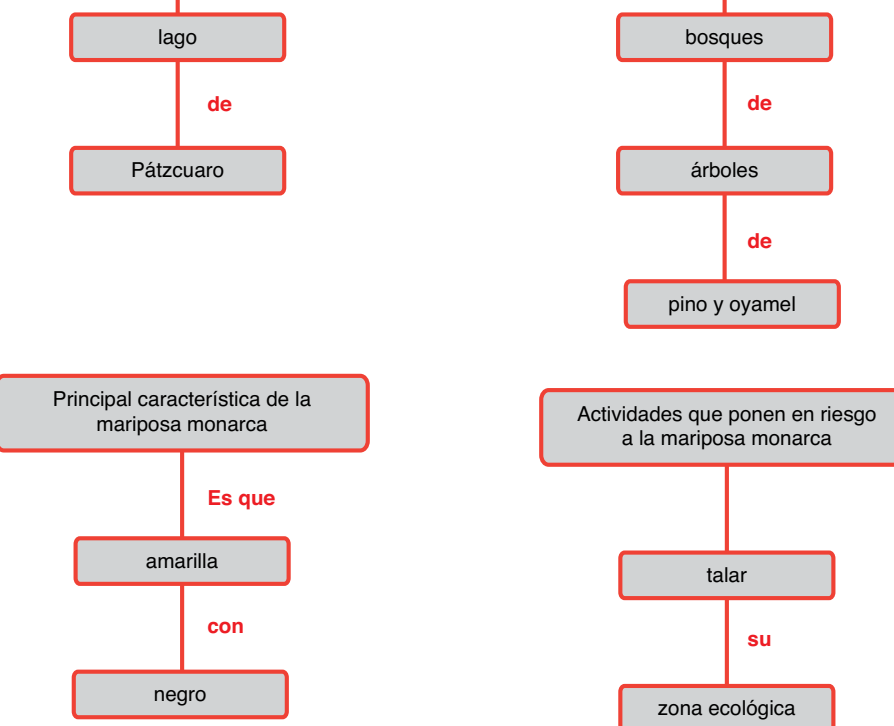

Actividades que ponen en riesgo a la mariposa monarca

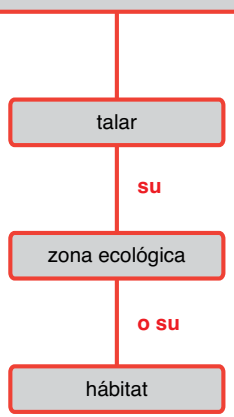

Esquema 2. 24 CBTIS pre (-)

Hábitat del pez blanco

Que es

Carnívoro
Hábitat de la mariposa monarca

Vive en los

Bosques templados

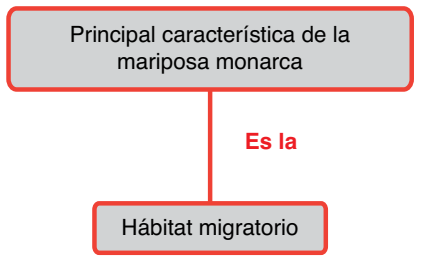

Actividades que ponen en riesgo a la mariposa monarca

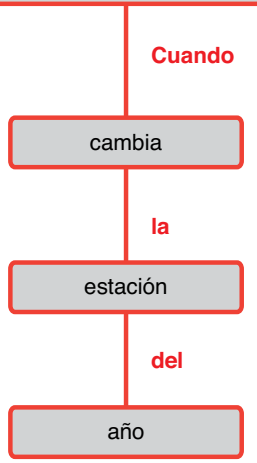


Sin embargo, los conceptos que presentan nulo cambio conceptual se distribuyen en su mayoría en el nivel explicativo, ello los hace imprescindibles para el establecimiento de relaciones con el resto de conceptos ecológicos básicos, y coincide con las dificultades encontradas por Guillén (1995), en el sentido de la falta de concepciones explicativas en alumnos del nivel bachillerato.

En general, sólo el 50\% de los reactivos correspondientes a los conceptos básicos de ecología muestran asimilación cognitiva, ya sea con conocimiento previo o luego de la intervención docente. La organización conceptual en promedio para ambos planteles se ubica dentro de un margen que va del marco nocional al referencial, lo cual corresponde a una organización conceptual baja, con base en la clasificación del Modelo de Análisis

\section{Referencias}

Alucema, M. A. (2001), "Procesos estratégicos en la construcción de contenido lógico-conceptual sobre la teoría evolutiva moderna en el nivel universitario", en M.A. Campos, Construcción de conocimiento y educación virtual, México, UNAM.

Barraza, L. y A. D. Cuarón (2004), "How values in education affect children's environmental knowledge", en Journal of Biological Education, vol. 39, núm.1, pp. 18-23.

Berger, P. L. y T. Luckmann (2001), La construcción social de la realidad, Buenos Aires, Amorrortu.

Bermudez, G. y A. L. de Longhi (2008), "La educación ambiental y la ecología como ciencia. Una discusión necesaria para la enseñanza", en Revista Electrónica de Enseñanza de las Ciencias, vol. 7, núm. 2, pp. 275-297.

Burns, T. P. (1992), "Ecosystem: a powerful concept and paradigm for ecology", en Bulletin of the Ecological Society of America, 73 (1), pp. 39-43.

Campos, M. A. y L. Cortés R. (2005), "El contenido epistemológico del conocimiento de estudiantes de
Proposicional (MAP) (Campos y Gaspar, 1996). Esto manifiesta la dificultad para el manejo de los conceptos considerados como fundamentales para la comprensión de los procesos ecológicos.

Los estudiantes de la muestra carecen de la estructura conceptual ecológica básica suficiente, sustentada en un conocimiento científico; esto a pesar de la evidencia mínima de cambio conceptual observado en algunos conceptos. Esto significa que predomina un conocimiento tácito sobre un conocimiento científico. Para que el conocimiento pueda considerarse como científico debe contener un explícito y amplio contenido categorial, una compleja red de conexiones lógicas con referencias factuales adecuadas y formas de elaboración, algo que no logró demostrarse en esta investigación.

biología en secundaria", en M. A. Campos (coord.), Construcción de conocimiento en el proceso educativo, México, UNAM-CESU/Plaza y Valdés.

Campos, M. A. y S. Gaspar H. (1996), "El modelo de análisis proposicional: un método para el estudio de la organización lógico-conceptual del conocimiento", en M. A. Campos y R. Ruiz, Problemas de acceso al conocimiento y enseñanza de las ciencias, México, UNAM.

Campos, M. A. y S. Gaspar H. (2004), "Análisis de la intertextualidad y la argumentación en el contexto educativo. Elementos teórico-metodológicos", en Revista Mexicana de Investigación Educativa, vol. 9, núm. 21, pp. 424-449.

Campos, M. A., L. Cortés y S. Gaspar (1999), "La Organización conceptual de estudiantes de secundaria sobre el concepto de biomoléculas", en Revista Mexicana de Investigación Educativa, vol. 3, núm. 7, pp. 27-77.

Cortés, L. (2005), "El tema de salud en secundaria: cómo construyen los estudiantes el conocimiento", 
en M. A. Campos (coord.), Construcción de conocimiento en el proceso educativo, México, UNAMCESU/Plaza y Valdés.

COSNET (2004), Modelo de la educación media superior tecnológica. Reforma curricular de la educación media superior tecnológica, México, COSNET.

Curiel, A. (2004), "Las preguntas clave para la educación ambiental en el nivel bachillerato", en Revista Educar, disponible en: <http://educar. jalisco.gob.mx/13/13Arturo.html> [Consulta: 12 de noviembre de 2009].

Degua, C. (2005), "El análisis del discurso en el aula: un estudio en el nivel medio superior desde la perspectiva sociocultural", en M. A. Campos (coord.), Construcción de conocimiento en el proceso educativo, México, UNAM-CESU/Plaza y Valdés.

De Posada, J. M. (2002), "Memoria, cambio conceptual y aprendizaje de las ciencias", en Revista Electrónica de Enseñanza de las Ciencias, vol. 1, núm. 2, pp. 92-113.

Fernández G., A. y E. Gumá D. (2001), "Un enfoque neurocognitivo de la conciencia", en V. M. Alcaraz R. y E. Gumá D., Texto de neurociencias cognitivas, México, E1 Manual Moderno.

Guillén, F. (1995), “¿Qué saben los estudiantes de secundaria sobre el tema de evolución?", en M. A. Campos (comp.), Problemas de acceso al conocimiento, México, IIMAS-UNAM.
Jiménez, M. P. (1989), "Los esquemas conceptuales sobre la selección natural: análisis y propuestas para un cambio conceptual", Tesis doctoral, Madrid, Universidad Complutense de Madrid.

Leff, E. (2000), "Pensar la complejidad ambiental", en E. Leff (coord.), La complejidad ambiental, México, Siglo XXI/UNAM.

Morin, E. (1996), "El pensamiento ecologizado", en Gazeta de Antropología, núm. 12, disponible en: <http://www.ugr.es/ pwlac/G12_01Edgar_ Morin.html> [Consulta: 15 de noviembre de 2009].

Novak, J. D. y D. Gowin (1988), Aprendiendo a aprender, Barcelona, Martínez Roca.

Ontoria, A., A. Ballesteros, C. Cuevas, L. Giraldo, I. Martín, A Molina, A. Rodriguez y U. Vélez (2000), Mapas conceptuales. Una técnica para aprender, Madrid, Narcea.

Oyama, K. (2002), "Nuevos paradigmas y fronteras en ecología”, en Revista Ciencias, núm. 67, pp. 20-31. Pozo, J. I. (2002), Teorías cognitivas del aprendizaje, Madrid, Morata.

Scheiner, A. M., A. J. Hudson y M. A. Vandermeulen (1993), "An epistemology for ecology", en Bulletin of the Ecological Society of America, 74 (1), pp. 17-21.

Torres O., S. (2006), Conceptos básicos de ecología en la escuela secundaria en Michoacán, México, Morelia, SEP/CONACYT/UMSNH.

\section{Cómo citar este artículo:}

Torres-Ochoa, Sergio-R. y Luis-Alfonso Cuevas-Novoa (2011), "Evaluación de adquisición de conocimientos de conceptos de ecología en estudiantes de bachillerato tecnológico en México", en Revista Iberoamericana de Educación Superior(RIES), México, ISSUE-UNAM/Universia, vol. II, núm. 3, pp. 130-151, http://ries.universia.net/index.php/ries/ article/view/80 [Consulta: fecha de última consulta]. 